\title{
Laparoscopy with nephroscopy in single-stage necrosectomy for the treatment of severe acute pancreatitis.
}

\author{
Jun-Jie Yin, Bei Lu, Zhong Jia, Yang Cai* \\ Department of Hepatopancreatobiliary Surgery, Hangzhou First People's Hospital, Nanjing Medical University, PR \\ China
}

\begin{abstract}
Background: Severe acute pancreatitis (SAP) is difficult to manage despite the availability of different endoscopic techniques. The most beneficial protocol remains unclear.

Objective: To explore the efficiency and safety of a combination of laparoscopy and nephroscopy in single-stage necrosectomy for the treatment of SAP.

Methods: A 57-year-old woman with SAP underwent microinvasive surgery (combination of laparoscopy and nephroscopy for first necrosectomy) along with dual-catheter drainage.

Results: The patient recovered uneventfully. She was followed up for 5 months. No surgery-related complications or other adverse events occurred. She was living a functional life at the time of this writing.

Conclusion: The combination of laparoscopy and nephroscopy is accessible and safe for single-stage surgical treatment of conditions involving the abdomen and retroperitoneum. This technique reduces healthcare costs and shortens the hospital stay. It is a clinically valuable technique for surgeons to learn.
\end{abstract}

Keywords: Laparoscopy, Necrotizing pancreatitis, Necrosectomy, Nephroscopy.

\section{Introduction}

Severe acute pancreatitis (SAP) is a critical illness mainly because of its complications and high mortality. Death frequently occurs due to systemic inflammatory response syndrome at the onset of SAP and peripancreatic abscess formation in the late stage of SAP. Peritoneal dialysis or hemodialysis is an effective technique for early therapy. Surgical drainage in all phases of the disease, especially via a minimally invasive route, is the key to successful treatment. Determination of the optimal operation timing and choice of appropriate strategy play a similarly important role. Although the use of endoscopic techniques has become widespread, the combination of laparoscopy and nephroscopy in a single surgery has rarely been reported. We herein report a case of SAP that was successfully treated by laparoscopy and nephroscopy.

\section{Case Report}

A 57-year-old woman with a 13-day history of frequent abdominal pain that had worsened in the past $12 \mathrm{~h}$ was admitted to our hospital. B-mode ultrasound examination revealed cholelithiasis accompanied by cholecystitis. Her blood amylase concentration was $1309 \mathrm{U} / \mathrm{L}$. Based on these primary findings, she was diagnosed with biliary pancreatitis. Physical examination revealed a body temperature of $37.2^{\circ} \mathrm{C}$, blood pressure of $121 / 81 \mathrm{mmHg}$, obvious upper abdominal tenderness, suspected rebound pain, and positive Murphy's sign. Computed tomography (CT) showed acute pancreatitis, abdominal effusion, and cholecystitis. Endoscopic retrograde cholangiopancreatography showed choledocholithiasis, which was treated by endoscopic sphincterotomy and endoscopic nasobiliary drainage. B-mode ultrasound-guided percutaneous drainage was then performed in the early stage of the patient's SAP, but her condition seemed to worsen.

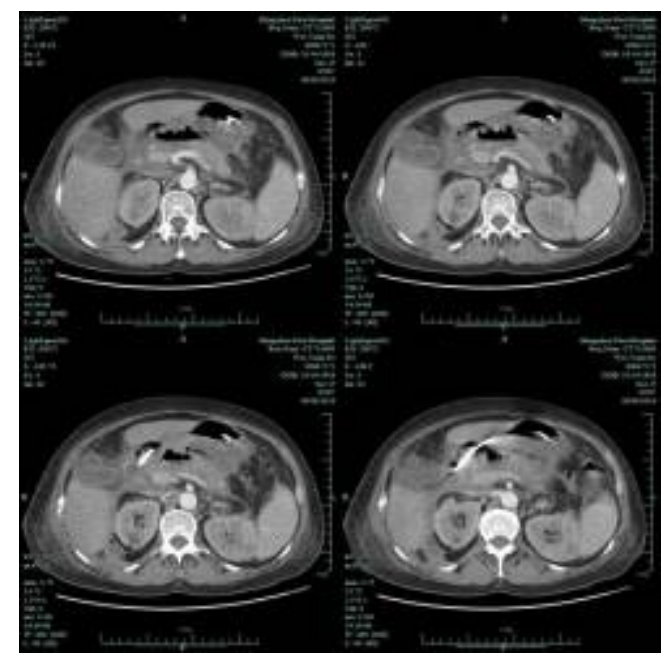

Figure 1. Computed tomography showed retroperitoneal gas accompanied by fluid within a pseudocyst of the pancreas. 


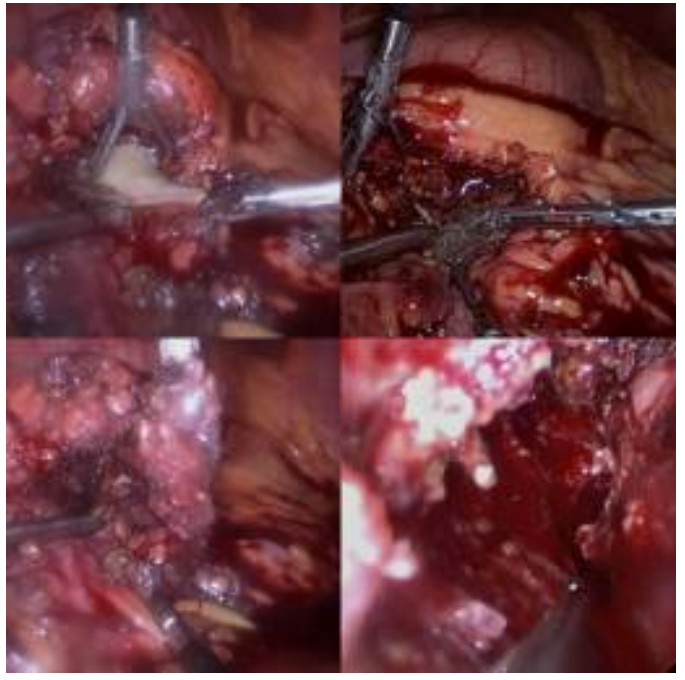

Figure 2. Pancreatic abscess drainage and cholecystectomy through a laparoscopic approach.

The patient's findings, including retroperitoneal gas and coexisting fluid in a pancreatic pseudocyst, were important signs to consider with respect to the possibility of infection (Figure 1). Therefore, the patient underwent a series of procedures including endoscopic nasobiliary drainage, percutaneous catheter drainage, pancreatic abscess drainage, and cholecystectomy through a laparoscopic approach and retroperitoneal necrosectomy through a percutaneous nephroscopic approach in a single surgery about 5 weeks later (Figure 2). During surgery, laparoscopic examination revealed a large amount of pus, extensive necrosis of the pancreas, and retroperitoneal fasciitis, while nephroscopic examination revealed a right retroperitoneal abscess and massive necrosis. After surgery, two sets of dual-catheters were placed in an appropriate peripancreatic position and in the retroperitoneal space to ensure adequate drainage. Continuous infusion of normal saline via a dual-catheter was then established. Reevaluation via contrast-enhanced CT of the pancreas 2 weeks after surgery revealed a significantly better outcome compared with the previous findings. About 1 month after surgery, a second-look operation via a nephroscopic approach through the sinus tract was successfully performed, eliminating the residual necrosis (Figure 3). The pus was redrained, the pancreatic necrosis was debrided, and the dual-catheter was readjusted. Similar work was conducted per 2 to 3 weeks until a small amount of necrosis was found in the retroperitoneal space. Therefore, the dual-catheter was gradually retracted until it was removed entirely. About 2 months later, contrast-enhanced CT of the abdomen showed only slight pancreatic necrosis, as expected (Figure 4). The patient was discharged uneventfully about 2 months after surgery. She was followed up for about 5 months on an outpatient basis, and no surgery-related complications or signs of relapse were found.

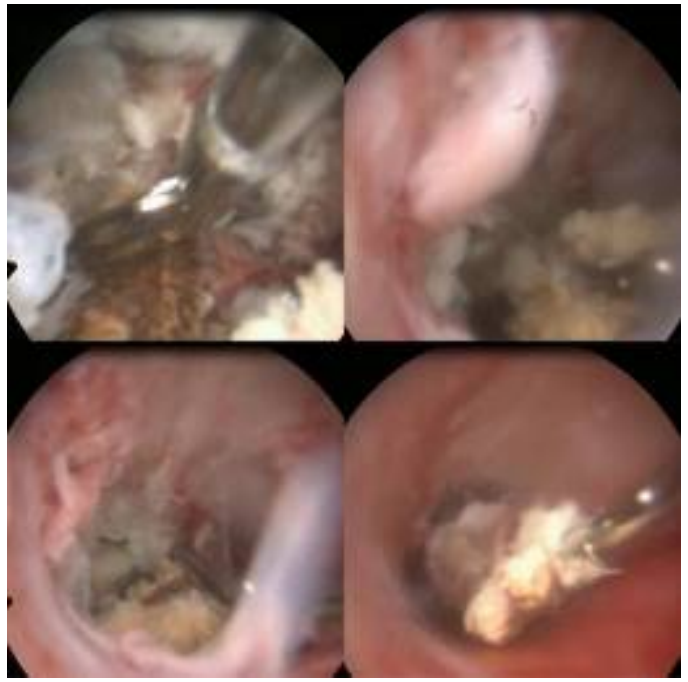

Figure 3. The second-look operation through a sinus tract with a nephroscopic approach was performed successfully.

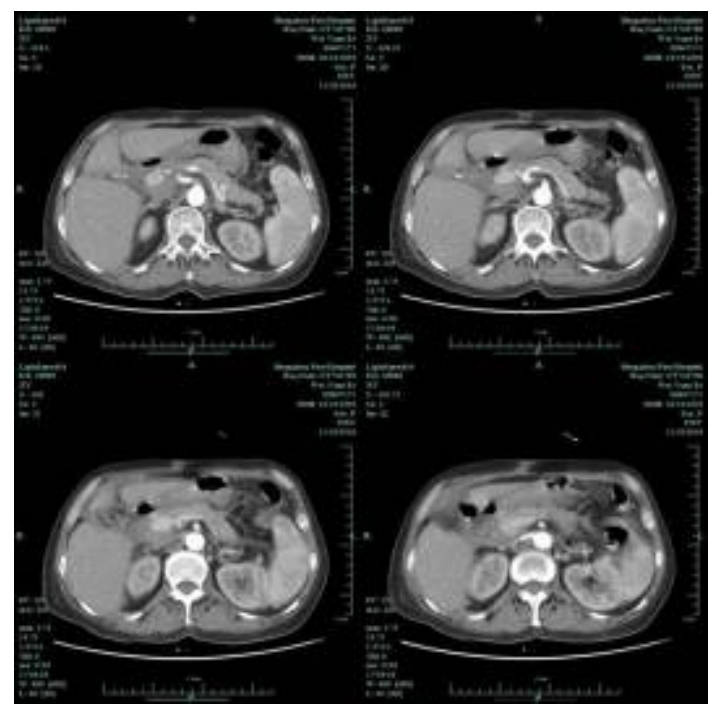

Figure 4. About 2 months later, contrast-enhanced computed tomography of the abdomen showed only slight necrosis of the pancreas, as expected.

\section{Discussion}

Infected necrotizing pancreatitis is one of the most serious complications of acute necrotizing pancreatitis. It often leads to multiple organ failure. The mortality rate is nearly $100 \%$ if no surgical interventions are applied [1]. Even with treatment, its complication and mortality rates are $20 \%$ to $25 \%$ and $43 \%$, respectively [2]. Only reoperation with the aim of eliminating the residual infected focus is recommendable [3]. However, reoperation is often challenging, particularly via an endoscopic approach, because many patients have a "frozen abdomen" characterized by extensive inflammation, tissue adhesion, and tissue edema. The risks of postoperative intestinal fistula, bleeding of tiny vessels, and superinfection are substantially high. 
Minimally invasive procedures such as percutaneous catheter drainage, laparoscopic debridement, and endoscopic surgery through a sinus tract have recently become prevalent worldwide [4]. Minimally invasive surgery has the advantages of less trauma, greater preservation of the internal and external secretory functions of the pancreas, easy postoperative management, and minimal postoperative pain. It is associated with fewer surgical complications and a lower mortality rate than open surgery [5]. Generally, the cure rate of percutaneous catheter drainage is only about $40 \%$ because pancreatic necrosis and peripancreatic fat necrosis are not easily liquefied [6]. Drainage is thus difficult to establish, increasing the risk of refractory infection and a long hospital stay.

Percutaneous nephroscopy for necrosectomy to treat SAP has been confirmed to be a reasonable and effective procedure since it was first reported by Carter et al. [7]. In 2009, the General Hospital of the Chinese People's Liberation Army began to improve this technique. They used gas instead of water to create the operative space to avoid the movement of water [8]. The prevalence of endoscopic surgery for SAP has rapidly increased $[9,10]$, and its safety and feasibility have been proven in selected cases. Compared with open surgery, the greatest advantages of endoscopy include less postoperative pain, faster recovery, and greater cosmesis. However, the nephroscopic orifice is too small for easy completion of necrosectomy; such an approach would require excessive time and energy. In addition, most patients must undergo a similar surgery several times. Biliary-related etiologies or abdominal problems cannot be resolved in a single surgery mainly because nephroscopy is restricted to the retroperitoneal space alone. Therefore, we combined a laparoscopic and nephroscopic approach in a single surgery.

A. Synchronous dual-endoscopy approach: This is a laparoscopic approach that aims to loosen the "frozen abdomen" and thus create the potential for necrosectomy to treat both necrotizing pancreatitis and necrotizing fasciitis. In addition, cholecystectomy and/or common bile duct exploration can be performed to resolve the etiology of biliaryorigin pancreatitis. Percutaneous nephroscopy, aiming to drain the retroperitoneal abscess, can then be performed to address extra-abdominal problems. The combination of two different endoscopic procedures can complement any shortcomings between them, providing benefits by their "overlapping effects." This also means that synchronous intra-abdominal and extra-abdominal operations are possible.

B. Dual-catheter drainage: A dual-catheter consists of three different sizes in diameter of tube (the biggest one is outside part, the middle one and the smallest one are inside part). The smallest one, which is used to dilute the pus and communicate various foci, is infused with normal saline. The middle one, which is used to drain necrotic tissue and pus, is connected to a negative-pressure device. The largest one is fixed to the other two to avoid their accidental slippage. In this way, adequate drainage is ensured.

C. Step-down trauma: Based on re-evaluation using contrastenhanced computed tomography of the abdomen every 2 to 3 weeks, re-operation for further debridement of necrotic tissue and re-adjustment of the dual-catheter during later more frequent debridements can be performed. The whole therapeutic period can be described as a "step-down" approach compared with the concept of the step-up approach [11]. The step-down approach includes a decreasing scale of necrosectomy and dual-catheter removal with 1 to $2 \mathrm{~cm}$ through the sinus tract under local anesthesia.

In our practice, the drainage tube is placed in the pancreatic bed along with the omental bursa, while the peritoneal dialysis tube is placed at the lowest position of the pelvis. After the operation, peritoneal lavage is performed for easy removal of pus. Although the laparoscopic technique is prioritized [12], it may also cause iatrogenic spread of infection and aggravate the patient's symptoms [13]. Laparoscopic resection of pancreatic necrosis has not been widely applied in practice and is not supported by bulk or randomized trials. The success rate is reportedly about $77 \%$, and the mortality rate is about $11 \%$. The main complications include pancreatic fistula formation, abdominal infection, and retroperitoneal infection [14]. With respect to the optimal timing of surgery, it is advisable to wait until 4 weeks after the onset of pancreatitis if possible. At this time, the patient's general condition has improved and stabilized. Furthermore, the border between the necrotic tissue and normal tissue may be easier to identify. A fibrous band or septum has begun to form. Surgery should be conducted if the retroperitoneal space contains gas to decrease the risk of colon penetration. If available, ultrasound- or CT-guided paracentesis should be carried out in a timely manner.

Any undesired violation of the procedure may result in bleeding. If resistance is felt when the necrotic tissue is grasped, the tissue should be freed. The goal of the first operation is to drain the pus by loosening the necrotic tissue. The necrotic tissue after loosening could be performed under local anesthesia. New double-catheterization cannulas were readjusted and replaced in each operation of the present case. Finally, the drainage tube in our patient was removed 22 weeks after disease onset. No bleeding, pancreatic fistula, or other complications occurred during her therapeutic course. No abdominal pain, fever, or other symptoms were found at her postoperative 5-month follow-up.

\section{Conclusion}

The combination of laparoscopy and nephroscopy within a single surgery can overlap the advantages of laparoscopy or nephroscopy alone. This technique may be a practical alternative choice for necrosectomy in the treatment of necrotizing pancreatitis because of its accessibility and flexibility. Notably, for high-risk patients, extensive necrosectomy by two endoscopic techniques may be counterproductive due to the potential of broad bleeding from tiny vessels, super-bacteremia, and undesired iatrogenic injuries. In our opinion, a dual-endoscopy approach is more suitable for lower-risk patients, mainly because of the lack of enough experience. The key to success is to hold scale of biting necrosis to be just perfect. 


\section{Acknowledgement}

This study was sponsored by the Hangzhou Science and Technology Program of Zhejiang Province.

\section{References}

1. Connoe S, Alexakis N, Raraty M. Early and late complications after necrosectomy. Surgery 2005; 137 : 499-505.

2. Lc Mee J, Paye F, Sauvanet A. Incidence and reversibility of organ failure in the course of sterile or infected necrotizing pancreatitis. Arch Surg 2001; 136: 1386-1390.

3. Fink D, Soares R, Matthews JB. History, goals, and tschnique of laparoscopic pancreatic necrosectomy. J Gastrointest Surg 2011; 15: 1092-1097.

4. Cirocchi R, Trastulli S, Desiderio J. Minimally invasive necrosectomy versus conventional surgery in the treatment of infected pancreatic necrosis: a systematic review and a meta-analysis of comparative studies. Surg Laparosc Endosc Percutan Tech 2013; 23: 8-20.

5. Raraty MG, Halloran CM, Dodd S. Minimal access retroperitoneal pancreatic necrosectomy: improvement in morbidity and mortality with a less invasive approach. Ann Surg 2010; 251: 787-793.

6. Bucher P, pugin F, Morel P. Minimally invasive necrosectomy for infected necrotizing pancreatitis. Pancreas 2008; 36: 113-119.

7. Carter RC, McKay CJ, Imrie CW. Percutaneous necrosectomy and sinus tract endoscopy in management of infected pancreatic necrosis: An initial experience. Ann Surg 2000; 232: 175-180.

8. Shouwang C, Pengfei W, Zhiwei L. Improvement and effect of retroperitoneoscopic necrosectomy for infected necrotizing pancreatitis. Chin J Hepatobiliary Surg 2012; 18: 439-441.

9. Luigiano C, Pellicano R, Fusaroli P. Pancreatic necrosectomy: an evidence-based systematic review of the levels of evidence and a comparison of endoscopic versus non-endoscopic techniques. Minerva Chir 2016; 71: 262-269.

10. Kumbhari V, Storm AC, Tieu AH. Percutaneous flexible endoscopic necrosectomy for a retroperitoneal abscess. Endoscopy 2014; 46: 340-341.

11. Van Santvoort HC, Besselink MG, Bakker OJ. A step-up approach or open necrosectomy for necrotizing pancreatitis. N Engl J Med 2010; 362: 1491-1502.

12. Navaneethan U, Vege SS, Chaf ST. Minimally invasivetechniques in pancreatic necrosis. Pancreas 2009; 38: 867-875.

13. Xin J, Huabo J, Yuliang T. Minimally invasive treatment of necrotizing panereatitis. Chin J Clinicians 2016; 10: 1627-1630.

14. Melman L, Azar R, Beddow K. Primary and overall success rates for clinical outcomes after laparoscopic, endoscopic, and open pancreatic cystgastrostomy for pancreatic pseudocysts. Surg Endosc 2009; 23: 267-271.

\section{*Correspondence to}

Yang Cai

Department of Hepatopancreatobiliary Surgery

Hangzhou First People’s Hospital

Nanjing Medical University

PR China 